\title{
Long non-coding RNA MEG3 functions as a competing endogenous RNA of miR-93 to regulate bladder cancer progression via $\mathrm{PI3K} / \mathrm{AKT} / \mathrm{mTOR}$ pathway
}

\author{
Xinrong Fan, Houfeng Huang, Zhigang Ji, Quanzong Mao \\ Department of Urology, Peking Union Medical College Hospital, Chinese Academy of Medical Sciences, Beijing 100730, China \\ Contributions: (I) Conception and design: Q Mao, X Fan; (II) Administrative support: Q Mao; (III) Provision of study materials or patients: H Huang; \\ (IV) Collection and assembly of data: X Fan; (V) Data analysis and interpretation: Z Ji; (VI) Manuscript writing: All authors; (VII) Final approval of \\ manuscript: All authors. \\ Correspondence to: Quanzong Mao. Department of Urology, Peking Union Medical College Hospital, Chinese Academy of Medical Sciences, \\ Shuaifuyuan 1, Wangfujing, Dongcheng District, Beijing 100730, China. Email: quanzong_mao@163.com.
}

Background: Maternally expressed gene $3(M E G 3)$ is a long non-coding RNA (lncRNA) and involved in
progression of various human tumors. However, its underlying regulatory mechanism in tumorigenesis of
bladder cancer (BC) remains unclear. To demonstrate effects of $M E G 3$ on BC cell proliferation and elaborate
its regulatory mechanism in BC.

Methods: Aberrant expressions of MEG3 and miR-93-5p were induced by cell transfection. The mRNA and protein expression were analyzed using qRT-PCR and western blot. Cell proliferation was examined by CCK-8 assay and EdU staining. The targeted regulation effect of $M E G 3$ on $m i R-93-5 p$ was confirmed by luciferase reporter assay. The number of LC3 punctated cells was detected by immunofluorescence. Xenograft mouse model was constructed for in vivo validation.

Results: $M E G 3$ was down-regulated with increased expression of $m i R-93-5 p$ in BC cells and tissues. Luciferase reporter assay showed that miR-93-5p was a direct target of $M E G 3$ and was negatively regulated by $M E G 3$. MEG3 overexpression inhibited cell proliferation and the expression of proliferation-, apoptosisand autophagy-related proteins. The activation of PI3K/AKT/mTOR pathway was also suppressed with elevated cell apoptosis. $m i R-93-5 p$ overexpression counteracted these results. In vivo experiments, we confirmed that miR-93-5p overexpression reversed the $M E G 3$ overexpression-mediated suppression on tumor growth and protein expression.

Conclusions: lncRNA MEG3 could function as a competing endogenous RNA of $m i R-93$ to regulate the tumorigenesis of $\mathrm{BC}$ via $\mathrm{PI} 3 \mathrm{~K} / \mathrm{AKT} / \mathrm{mTOR}$ pathway. The present research provided a new perspective to understanding the pathogenic mechanism of $\mathrm{BC}$, and an effective therapeutic target for $\mathrm{BC}$.

Keywords: Maternally expressed gene 3; long non-coding RNA; bladder cancer (BC); autophagy; miR-93-5p

Submitted Oct 30, 2019. Accepted for publication Jan 03, 2020.

doi: $10.21037 /$ tcr.2020.01.70

View this article at: http://dx.doi.org/10.21037/tcr.2020.01.70

\section{Introduction}

Bladder cancer (BC) is emerged as a highly prevalent malignancy associated with high morbidity and mortality and its incidence has increased over the last decades $(1,2)$. At present, various therapies including surgery, chemotherapy and radiation treatment have been widely applied in the therapy of BC (1). However, the advances in $\mathrm{BC}$ treatment have been greatly restricted in the past decades due to the lack of understanding of its pathogenic mechanism. Thus, it is urgent to elaborate the pathogenic mechanism of BC. Recently, long noncoding RNAs (lncRNAs) have emerged as the 
crucial regulators in the tumorigenesis and metastasis (3), which could function as the tumor suppressor or oncogene in the tumorigenesis (4).

Maternally expressed gene 3 (MEG3) as a lncRNA was reported to serve as a tumor suppressor in human cancer cell lines and tissues $(5,6) . M E G 3$ is expressed in various normal human tissues, however its expression is downregulated in the tumors and tumor cell lines (7). Previous study has demonstrated that $M E G 3$ overexpression could promote apoptosis and inhibit proliferation (8). Particularly, the $M E G 3$ knockdown was proved to regulate the proliferation and autophagy in BC cells (9). Therefore, we speculated that $M E G 3$ might have inhibitory effects in the development and $\mathrm{BC}$ progression and can act as potential drug in anti-tumor therapy. However, the underlying mechanism is still unclear.

It is well-accepted that lncRNAs sever as competing endogenous RNAs (ceRNAs) or sponges of microRNA (miRNAs) to indirectly regulate mRNAs expression by competing for binding to shared miRNAs (10). For example, Huang et al. indicated that $M E G 3$ impairs BC invasion by binding with miR-27a (11). Importantly, Zhang et al. previously reported that MEG3 binds with $m i R-93$ to inactivate PI3K/AKT pathway and thereby inhibits of glioma cell growth (12). Mammalian target of rapamycin (mTOR) is the downstream of PI3K/AKT pathway, which has effects in regulating differentiation, proliferation and controls whether a cell undergoes programmed cell apoptosis or autophagy (13). Thus, we speculated that $M E G 3$ may also affect the $\mathrm{BC}$ cell proliferation by targeting miR-93-5p and inactivating PI3K/AKT/mTOR pathway. Therefore, we aimed to demonstrate effects of $M E G 3$ on $\mathrm{BC}$ cell proliferation and elaborate whether $M E G 3$ regulate the BC progression by miR-93-5p and PI3K/AKT/mTOR pathway.

\section{Methods}

\section{Human samples}

Twenty pairs of surgical samples (BC tissues and normal tissues) were originally obtained from patients underwent surgical resection in Peking Union Medical College Hospital (Beijing, China) from 2017 to 2018. Patients who not received chemotherapy and radiotherapy before surgical resection were eligible for enrollment. Samples were frozen in liquid nitrogen and stored in the $-80{ }^{\circ} \mathrm{C}$ refrigerator until used. This study was approved by Ethics Committee of Peking Union Medical College Hospital (approval number: SYXK(Beijing)2018-0027). Informed consents were obtained from each patient before operation.

\section{Cell lines and culture}

Four BC cell lines (T24, UMUC3, 5637 and BIU87) and normal bladder epithelial SV-HUC-1 cell lines were originally obtained from Shanghai Institutes for Biological Sciences (Shanghai, China). Cells were grown in RPMI1640 medium supplementing with $10 \%$ heat-in-activated fetal bovine serum (Gibco, USA), $100 \mu \mathrm{g} / \mathrm{mL}$ streptomycin and $100 \mathrm{U} / \mathrm{mL}$ penicillin. Subsequently, cell lines were maintained in an incubator at $37^{\circ} \mathrm{C}$ with $5 \% \mathrm{CO}_{2}$.

\section{$R N A$ extraction and $q R T-P C R$}

Trizol reagent (Takara, Japan) was used to extract total RNA from cell lines and tissues samples. Reverse Transcriptase Kit was used to reversely transcribe RNA into cDNA (Takara, China). Then, mRNA expression was detected by SYBR Premix Ex Taq (Takara, China) on the ABI 7300 system (Applied Biosystems, USA). The relative expression of mRNAs was evaluated by $2^{-\Delta \Delta C t}$ method, using GAPDH and small nuclear RNA U6 as the internal control for miR93-5p and MEG3, respectively. PCR primer sequences were showed in Table 1.

\section{Cell transfection}

Cell transfection was performed using Lipofectamine 2000 (Invitrogen, Canada). Recombinant lentiviral vector carrying lncRNA $M E G 3$ or short hairpin RNAs (shRNA)$M E G 3$ were constructed to induce $M E G 3$ up-regulation or down-regulation as previously described (12). The miR93-5p mimics were obtained from Genepharma (Shanghai, China) to induce miR-93-5p up-regulation. BIU87 cells were transfected with lentiviral vector lncRNA $M E G 3$ and/ or $m i R-93-5 p$ mimics, respectively. Untransfected cells were used as a blank control.

\section{Luciferase reporter assay}

The MEG3 3'-UTR (WT) of the Renilla luciferase gene was cloned into psiCHECK2 dual-luciferase vector (Promega, USA). After 24 hours, cells $\left(1 \times 10^{5}\right.$ cells/well) were co-transfected with WT or MUT luciferase constructs $(0.4 \mathrm{ng} / \mu \mathrm{L})$ and $m i R-93-5 p$ mimics by Lipofectamine 2000 
Table 1 PCR primer sequences of target genes

\begin{tabular}{lcc}
\hline Gene & Forward & Reverse \\
\hline miR-93-5p & 5'-CAAAGTGCTGTTCGTGCAGGTAG-3' & 5'-GGATCCGACGGCTGGGTCTTCTCAGA-3' \\
U6 & 5'-TGCGGGTGCTCGCTTCGGCAGC-3' & 5'-GGATCCGACGGCTGGGTCTTCTCAGA-3' \\
GAPDH & 5'-CTGGGCTACACTGAGCACC-3' & 5'-AAGTGG TCGTTGAGGGCAATG-3' \\
MEG3 & 5'-CTGCCCATCTACACCTCACG-3' & 5'-CTCTCCGCCGTCTGCGCTAGGGGCT-3' \\
\hline
\end{tabular}

(Invitrogen, USA). After 72 hours transfection, Renilla and Firefly luminescence were analyzed.

\section{Cell viability assay and apoptosis assay}

Cell Counting Kit-8 (CCK-8, Dojindo Laboratories, Japan) was used to evaluate the cell viability. The miR-93-5p mimics or/and LncRNA-MEG3, or their negative control respectively transfected BIU87 cells. After 48 hours transfection, cells at a density of $5 \times 10^{3}$ were seeded into 96-well plates and then incubated for 1-4 days, respectively. After incubation, CCK-8 solution were added and then incubated for another 2 hours at $37^{\circ} \mathrm{C}$. The absorbance was measured at $450 \mathrm{~nm}$.

Cell apoptosis was measured by the flow cytometry assay. After transfection and incubation, cells were double-labeled with Annexin V-fluorescein isothiocyanate and propidium iodide apoptosis detection kits (eBioscience, USA). Then, cells were analyzed immediately in a BD FACSCalibur flow cytometer (BD Biosciences, USA).

\section{Western blot assays}

Primary antibodies to Ki67, PCNA, Caspase-3, cleaved Caspase-3, Caspase-9, cleaved Caspase-9, LC3II, LC3I, Beclin1, p53, Akt, p-Akt, mTOR, p-mTOR, Ulk, p-Ulk and GADPH (endogenous control) were used in the western blot assay. All primary antibodies were purchased from Abcam Cambridge, UK.

Total protein lysates were isolated by RIPA lysis buffer. Firstly, isolated protein was separated by sodium dodecyl sulfate-polyacrylamide gel electrophoresis (SDS-PAGE) and transferred onto a polyvinylidene fluoride (PVDF) membrane (Millipore, USA). PVDF membrane was then blocked by an incubation in phosphate-buffered saline (PBS) for $2 \mathrm{~h}$ at $37^{\circ} \mathrm{C}$. Subsequently, PVDF membranes were incubated with specific primary antibodies separately at $4{ }^{\circ} \mathrm{C}$ overnight. GAPDH was used as internal control. PVDF membrane were stained with the corresponding HRP- conjugated secondary antibodies (Abcam, UK), followed by visualized with enhanced chemiluminescence (ECL) system (GE Health-care, USA), and analyzed using Chemi-Doc XRS (Bio-rad, USA).

\section{5-ethynyl-2'-deoxyuridine (EdU) staining}

Cells were treated with EdU $(50 \mu \mathrm{mol} / \mathrm{L})$ and then fixed in $4 \%$ paraformaldehyde for $10 \mathrm{~min}$ at room temperature. Cells were permeabilized with TritonX-100 at $37{ }^{\circ} \mathrm{C}$ for 30 min after being washed PBS twice. Cells were then reacted with Apollo reaction cocktail $(1 \times, 100 \mu \mathrm{L})$ for $30 \mathrm{~min}$, and stained with Hoechst $33342(5 \mu \mathrm{g} / \mathrm{mL}, 100 \mu \mathrm{L})$ for $30 \mathrm{~min}$. Finally, cells were visualized under a fluorescent microscope.

\section{Immunofluorescence}

Cells were cultured to $70 \%$ confluence, washed twice, and fixed with $4 \%$ paraformaldehyde at room temperature for 15 min. After blocking with $1 \%$ bovine serum albumin, cells were incubated with primary antibody against LC3 (Abcam, ab58610). Then, cells were washed with PBS, and stained with secondary antibody to conjugated to HRP at room temperature for $50 \mathrm{~min}$. Cells were visualized under a Leica TCS SP II confocal laser scanning microscope (Leica, USA).

\section{Xeno-graft mouse model}

Forty male specific pathogen-free athymic nude mice (weighing 20-25 g, aged 6-8 weeks) were obtained from Shanghai National Center for Laboratory Animals (Shanghai, China). Mice were housed under specific pathogen-free conditions at humidity of $25 \pm 2{ }^{\circ} \mathrm{C} / 60 \% \pm 5 \%$, 12 hours light/dark cycle, free access to food and water. Animal experiment was performed in accordance with the National Institutes of Health Guide for the Care and Use of Laboratory Animals. 

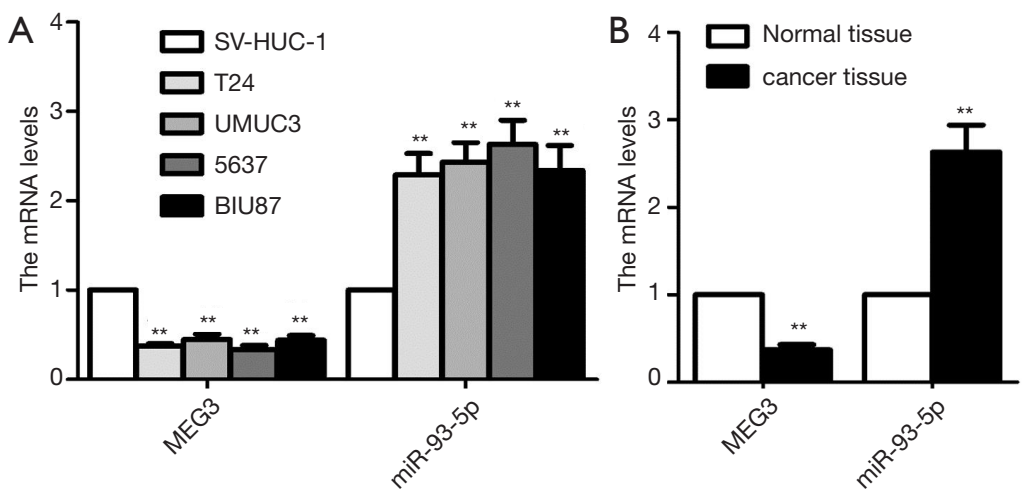

Figure 1 Differential expression levels of MEG3 and miR-93-5p in BC cell lines and tissues. (A) The relative expression levels of MEG3 and miR-93-5p in BC cell lines was measured by qRT-PCR, using normal SV-HUC-1 cells as control; (B) the relative expression levels of MEG3 and miR-93-5p was detected in BC tissues and normal tissues (control) by qRT-PCR. Data were presented as the mean \pm standard error and obtained from three independent experiments. ${ }^{* *}, \mathrm{P}<0.01$ compared to control.

Thirty SPF nude mice were subcutaneously injected with $1 \times 10^{7}$ BIU 87 cells transfected with $\operatorname{lncRNA}-M E G 3$ or/and miR-93-5p mimics randomly. Another ten mice were used as blank control. Then, the volume of tumor nodules was detected using external caliper every 6 days. The following formula was used to calculate tumor volumes: Volume $=$ (width + length) $/ 2 \times$ width $\times$ length $\times 0.5236$. After 30 days, all mice were sacrificed for immunohistochemistry assay. Tumor tissue was sectioned and fixed in $10 \%$ formalin for 48 hours, followed by paraffin embedded. Then, the tissue section was incubated with primary antibody anti Ki-67 (Dako, 1:50 dilution) at $4{ }^{\circ} \mathrm{C}$ for 12 hours and anti-Caspase 3 (Dako, 1:50 dilution). Secondary antibody streptavidinHRP-conjugated (Santa Cruz, CA, USA) was stained for 1 hour subsequently.

\section{Statistical analysis}

All statistical analyses were analyzed by SPSS 16.0 (IBM SPSS Inc., USA). All experiments were repeated in at least triplicate. Data are presented as mean \pm standard deviation (SD). Comparison analysis was performed by Student's $t$-test and one-way analysis of variance (ANOVA) when appropriate. The $\mathrm{P}$ value less than 0.05 was considered as statistically significant.

\section{Results}

\section{Differential expression of MEG3 and miR-93-5p in BC cells and tissues}

To evaluate the role of $M E G 3$ and $m i R-93-5 p$ in $\mathrm{BC}$ progression, $\mathrm{qRT}-\mathrm{PCR}$ was used to measure the expression in $\mathrm{BC}$ cell lines and tissues respectively. MEG3 was downregulated in all BC cells (T24, UMUC3, 5637 and BIU87) compared to SV-HUC-1 cell (all $\mathrm{P}<0.01$, Figure $1 A$ ). Conversely, $m i R-93-5 p$ was up-regulated in $\mathrm{BC}$ cell lines compared with SV-HUC-1 cell (all $\mathrm{P}<0.01$, Figure $1 A$ ). For the expression in $\mathrm{BC}$ cells, $M E G 3$ expression level was highest while miR-93-5p level was lowest in BIU87 cells compared to other three cell lines (Figure 1A). Consistently, MEG3 was significantly down-regulated and $m i R-93-5 p$ was overexpressed in $\mathrm{BC}$ tissues compared to normal tissues $(\mathrm{n}=20$ in each group, Figure $1 B)$. Overall, these results suggested the MEG3 and miR-93-5p may participate in BC progression. In present study, BIU87 cell line was used to perform the subsequent experiments since it had the highest $M E G 3$ and lowest $m i R-93-5 p$ expression level.

\section{MiR-93-5p is a direct target of MEG3}

Subsequently, MEG3 was knocked down or overexpressed by expressing shRNA-MEG3 or LncR-MEG3 in BIU87 cell line to confirm the function of $M E G 3$, respectively. As expected, the mRNA expression of MEG3 was significantly up-regulated or down-regulated compared to the control (Figure 2A). Furthermore, the miR-93-5p expression was significantly reduced by $M E G 3$ overexpression, while enhanced by MEG3 knock-down (Figure $2 A$ ), indicating that $m i R-93-5 p$ expression may be regulated by MEG3. Thereby, to verify the regulatory mechanism of $M E G 3$ and $m i R-93-5 p$, a bioinformatics analysis was performed in the RegRNA 2.0 databases (http://regrna2.mbc.nctu.edu. 


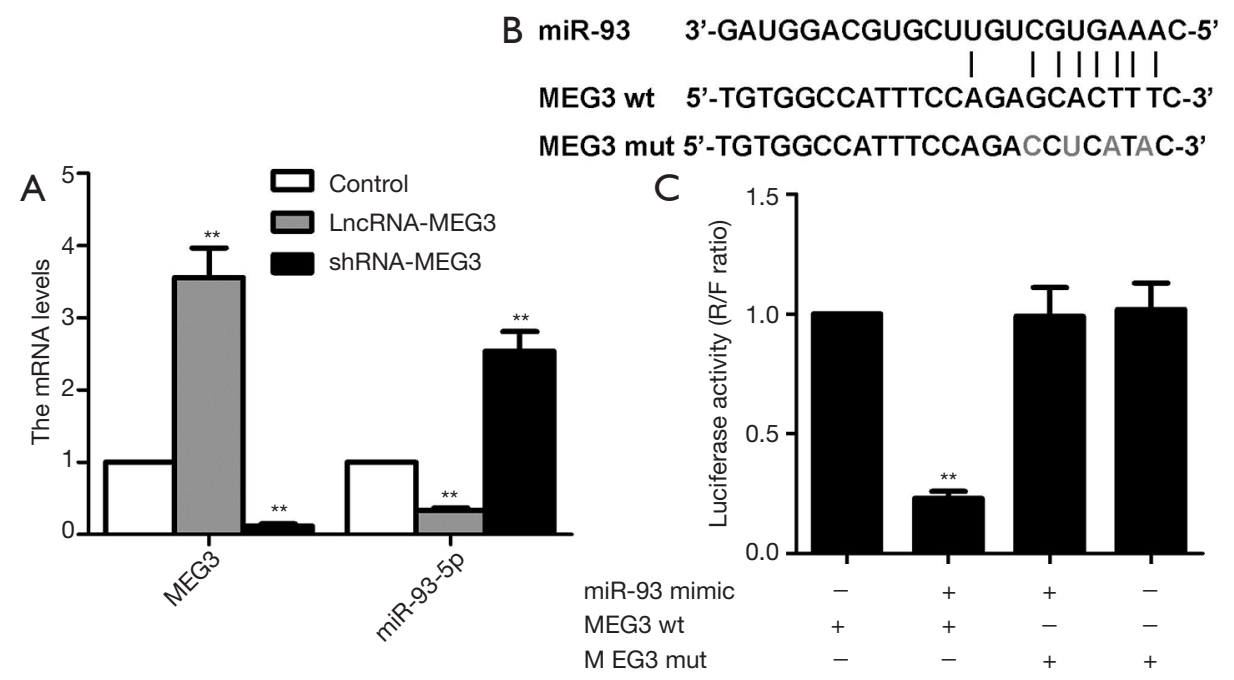

Figure 2 MiR-93-5p is a direct target of MEG3 and negatively regulated by MEG3. (A) Recombinant lentiviral vector carrying LncRNAMEG3 or MEG3-shRNA were transfected into BIU87 cells to induce MEG3 up-regulation or down-regulation. The mRNA expression levels of MEG3 and miR-93-5p were measured by qRT-PCR; (B) alignment of MEG3 with miR-93-5p 3'-UTR sequences; (C) relative luciferase activity in BIU87 cells co-transfected with miR-93-5p mimic and luciferase reporters containing wild type or mutated type MEG3 target sites. Data were presented as the mean \pm standard error and obtained from three independent experiments. ${ }^{* *}, \mathrm{P}<0.01$ compared to control.

$\mathrm{tw} /$ ) for its potential target sites. As shown in Figure $2 B$, the 3'UTR of $m i R-93-5 p$ harbors a consequential binding site for $M E G 3$, indicating that $m i R-93-5 p$ may be the potential regulatory target of $M E G 3$. Then luciferase reporter assay further demonstrated that relative luciferase activity was inhibited in BIU87 cell line co-transfected with miR-93$5 p$ and $M E G 3$ (Figure 2C). Overall, these results suggested that $m i R-93-5 p$ is a direct target of $M E G 3$, and negatively regulated by $M E G 3$.

\section{MEG3 overexpression inhibited the tumorigenesis of $B C$ cells by targeting miR-93-5p}

To investigate the potential molecular mechanism of $M E G 3$ and $m i R-93-5 p$ in BC progression, LncRNA-MEG3 and/ or miR-93-5p mimics were transfected into BIU87 cell line. CKK-8 assay demonstrated that the cell proliferation was significantly inhibited by $M E G 3$ up-regulation, while the miR-93-5p overexpression reversed the MEG3 overexpression-mediated suppression on cell proliferation (Figure $3 A$ ). Next, the expression of proliferation-related proteins including $\mathrm{Ki} 67$ and proliferating cell nuclear antigen (PCNA) were measured. As shown in Figure $3 B$ and $C$, MEG3 overexpression suppressed the expression levels of Ki67 and PCNA, while co-transfection with LncRNA-
$M E G 3$ and $m i R-93-5 p$ mimics significantly enhanced the MEG3 overexpression-mediated suppression on protein expression. EdU staining was performed to further detect the cell proliferation. Consistently, MEG3 overexpression significantly decreased the rate of EdU positive cells, while co-transfection with LncRNA-MEG3 and miR-93$5 p$ mimics evidently abrogated this effect (Figure 3D,E). Conversely, MEG3 overexpression significantly promoted cell apoptosis, while co-transfection with LncRNA-MEG3 and $m i R-93-5 p$ mimics abrogated this effect (Figure 3F,G). Meanwhile, western blot assay revealed that enhanced expression of MEG3 significantly promoted the expression of pro-apoptotic proteins (cleaved caspase- 3 and caspase-9), while miR-93-5p mimics reversed this effect (Figure 3H,I). Overall, these results suggested that MEG3 may function as a tumor suppressor gene in $\mathrm{BC}$, and $m i R-93-5 p$ function as an oncogene. Besides, MEG3 overexpression inhibited the tumorigenesis of $\mathrm{BC}$ cell lines by targeting $m i R-93-5 p$.

\section{MEG3 overexpression inbibited autophagy and the activation of PI3K/AKT/mTOR patbway by targeting $m i R-93-5 p$}

To explore whether MEG3 could regulate cell autophagy and PI3K/AKT/mTOR pathway by targeting $m i R-93-5 p$ in 

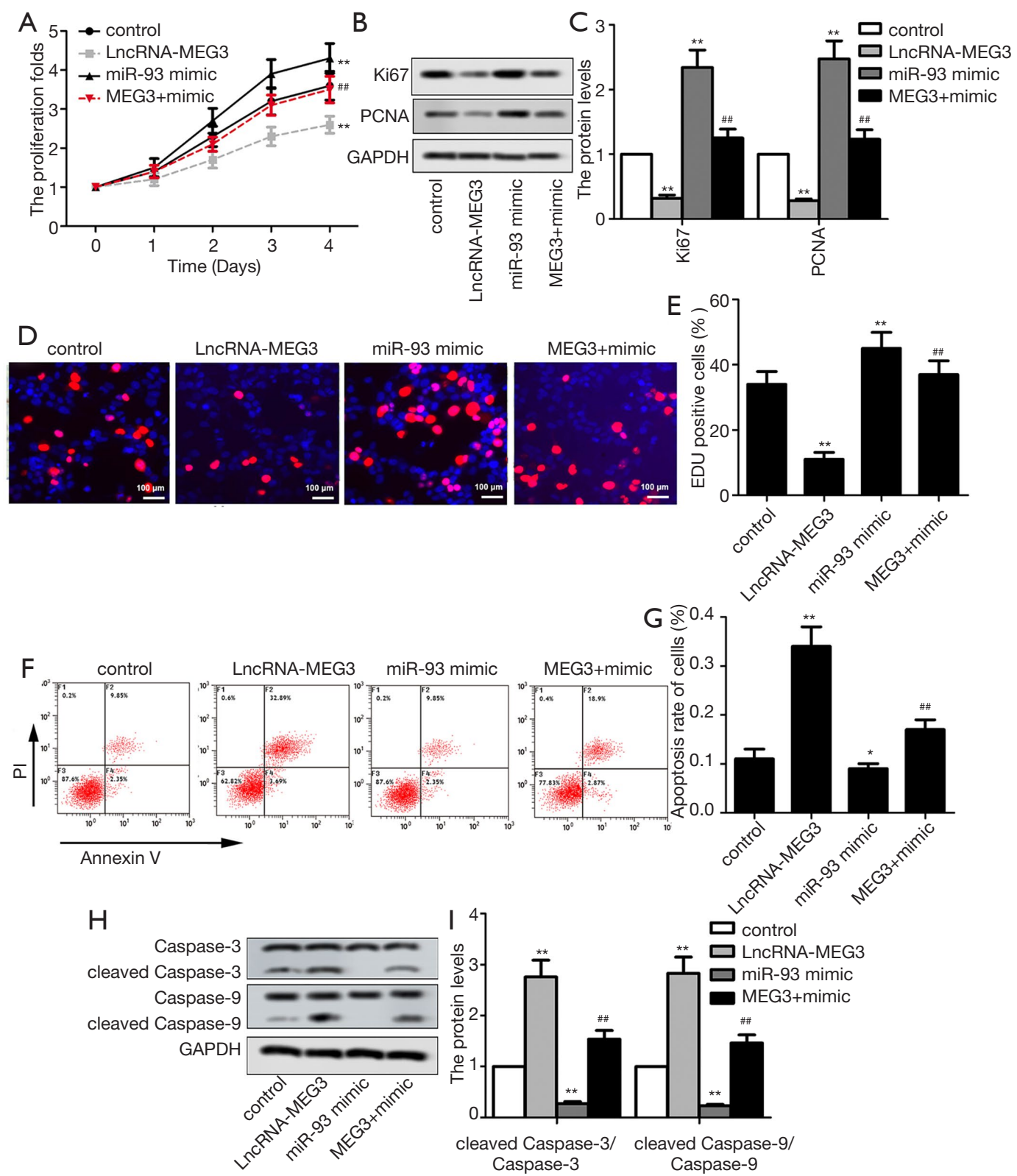

Figure 3 MEG3 overexpression inhibited the tumorigenesis of BC cell lines by targeting miR-93-5p. (A) Cell proliferation was measured by CCK-8 assay from day 1 to day 4 after cell transfection; (B,C) Western blot assay was performed to detect the proteins expression of Ki67 and PCNA in transfected BIU87 cells; (D,E) EdU positive cells after transfection were measured by EdU staining; (F,G) flow cytometry assay was conducted to analyze the cell apoptosis of transfected BIU87 cells; (H,I) Western blot assay was performed to detect pro-apoptotic proteins in transfected BIU87 cells. **, $\mathrm{P}<0.05$ compared to control; ${ }^{\#}, \mathrm{P}<0.05$ compared to BIU87 cells transfected with LncRNA-MEG3. 


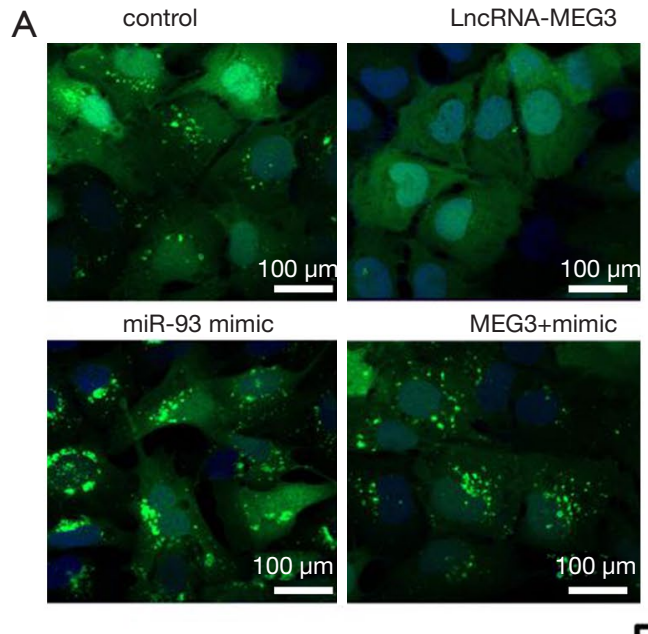

B
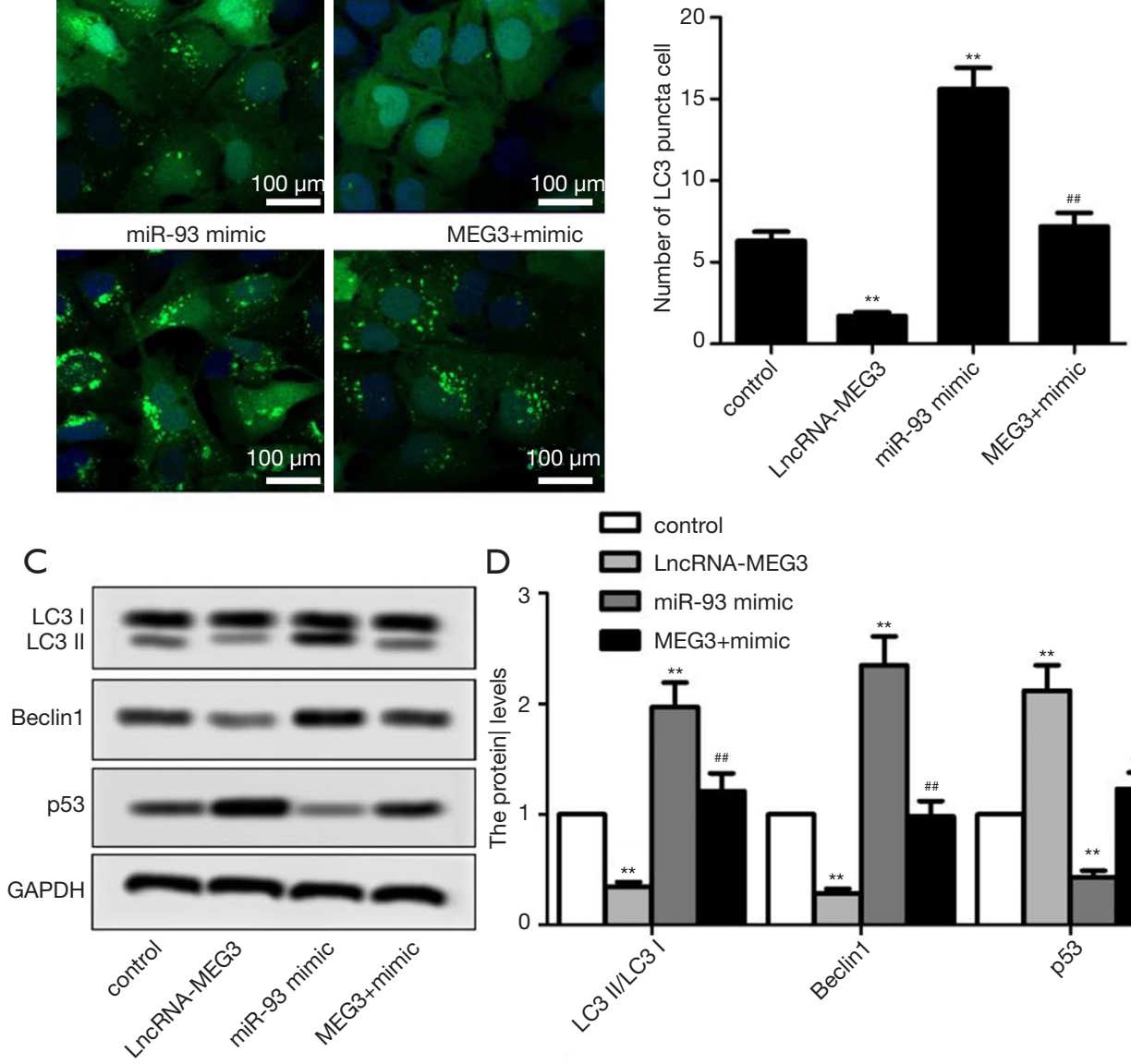

D LncRNA-MEG3
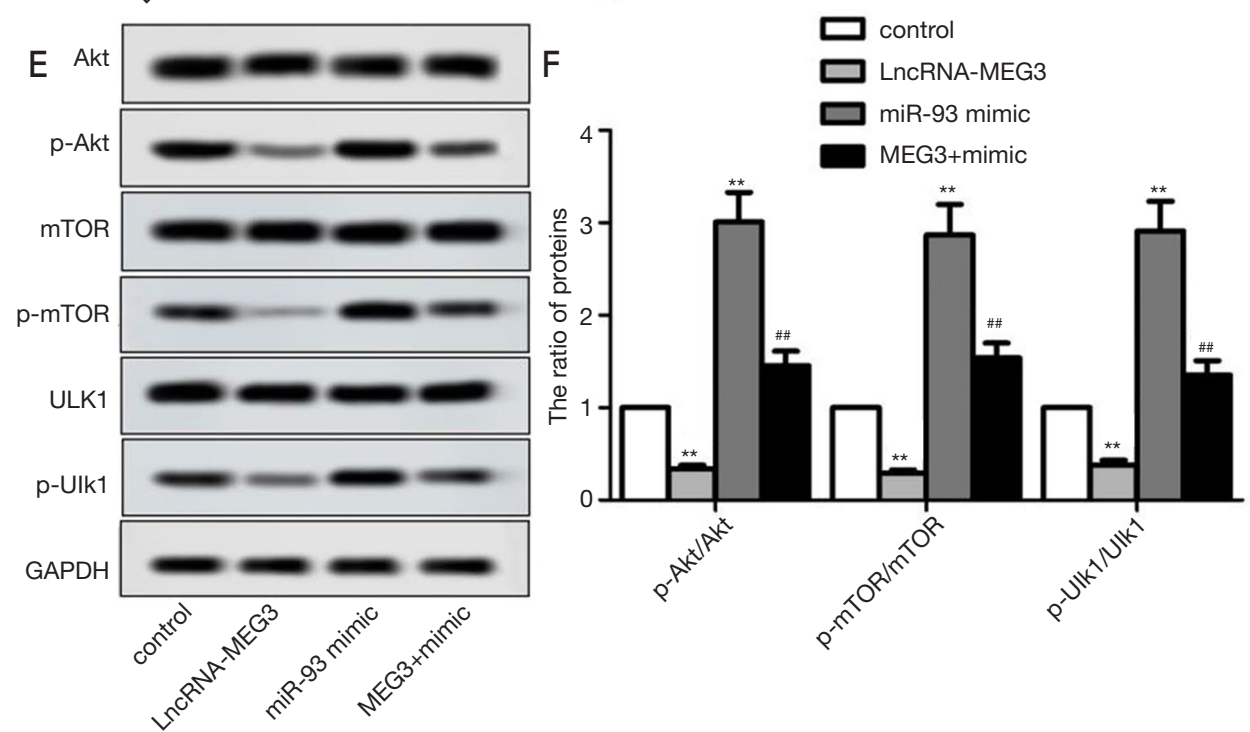

Figure 4 MEG3 overexpression regulated autophagy and the activation of PI3K/AKT/mTOR pathway by targeting miR-93-5p. (A,B) Immunofluorescence was used to detect the number of LC3 punctated cells after transfection; (C,D,E,F) Western blot was performed to evaluate the protein levels of autophagy-related proteins and in the expression level of $\mathrm{PI} 3 \mathrm{~K} / \mathrm{AKT} / \mathrm{mTOR}$ pathway. ${ }^{* *}$, $\mathrm{P}<0.05$ compared to control; "\#, $\mathrm{P}<0.05$ compared to BIU87 cells transfected with LncRNA-MEG3. 
$\mathrm{BC}$ cells, we measured the expression of autophagy-related proteins and proteins in $\mathrm{PI} 3 \mathrm{~K} / \mathrm{AKT} / \mathrm{mTOR}$ pathway in transfected BIU87 cells. Immunofluorescence presented a significant reduction in the number of LC3 punctated cells after transfection with LncRNA-MEG3, but miR-93$5 p$ mimics completely reversed the result (Figure $4 A, B$ ). With regard to the expression of autophagy-related proteins, MEG3 overexpression significantly suppressed the autophagy-related proteins (LC3II/LC3I, Beclin1 and P53), while $m i R-93-5 p$ mimics reversed the result (Figure 4C,D). Similarly, MEG3 overexpression decreased the rate of p-Akt/Akt, p-mTOR/mTOR and p-Ulk/Ulk, which was reversed by $m i R-93-5 p$ up-regulation (Figure 4E,F). Overall, these results indicated $M E G 3$ overexpression could inhibit autophagy and the activation of $\mathrm{PI} 3 \mathrm{~K} / \mathrm{AKT} / \mathrm{mTOR}$ pathway by targeting $m i R-93-5 p$ in $\mathrm{BC}$ cell lines.

\section{MEG3 overexpression inhibited tumorigenesis in vivo by targeting the miR-93-5p-mediated PI3K/AKT/mTOR patbway}

To provide in vivo evidence for the regulatory mechanism of $M E G 3$, xeno-graft mouse model was constructed to confirm the relationship between $M E G 3$ and $m i R-93-5 p$. As shown in Figure $5 A$, the tumor volume was significantly reduced after $M E G 3$ overexpression, while the overexpression of miR-93$5 p$ reversed the MEG3 overexpression-mediated suppression on tumor volume. IHC staining using Ki67 antibody showed that MEG3 overexpression suppressed the expression levels of Ki67, while co-transfection with LncRNA-MEG3 and $m i R$ 93-5p mimics significantly reversed this result (Figure $5 B, C$ ). Inversely, $M E G 3$ overexpression enhanced the caspase-3 expression, which was also reversed by $m i R-93-5 p$ mimics (Figure 5D,E). Finally, we explored the influence of miR-93$5 p$ on autophagy-related proteins and $\mathrm{PI} 3 \mathrm{~K} / \mathrm{AKT} / \mathrm{mTOR}$ pathway in vivo. Similarly with the in vitro experiments, $M E G 3$ overexpression significantly decreased the rate of LC3II/LC3I, p-Akt/Akt, p-mTOR/mTOR and p-Ulk/Ulk, and also reduced the expression levels of Beclin1 and P53. However, miR-93-5p mimics completely reversed these results (Figure 5F,G,H,I). Together, these results indicated that MEG3 overexpression inhibited tumorigenesis in vivo by targeting miR-93-5p-mediated PI3K/AKT/mTOR pathway.

\section{Discussion}

To our knowledge, this is the first report focused on the regulatory mechanism of lncRNA $M E G 3$ and $m i R-93$ in BC progression. The present study demonstrated that lncRNA $M E G 3$ functioned as a ceRNA of $m i R-93$ to suppress autophagy and induce cell apoptosis by inactivating PI3K/ $\mathrm{AKT} / \mathrm{mTOR}$ pathway in the progression of $\mathrm{BC}$.

We verified MEG3 was down-regulated in $\mathrm{BC}$ cell lines and tissues while $m i R-93-5 p$ was up-regulated, indicating that lncRNA MEG3 and miR-93-5p may be involved in the BC progression. Previously, many studies have reported the down-regulation of lncRNA $M E G 3$ and up-regulation of miRNAs in BC cell lines and tissues $(14,15)$. Similarly, lncRNA $M E G 3$ was decreased in many other tumors, such as esophageal squamous cell cancer (16), gastric cancer (7), lung cancer (17), and prostate cancer (18). These above evidences showed that lncRNA MEG3 may be an important regulatory factor of the tumorigenesis in various type of tumor.

lncRNAs acts as a sponge, could sequester miRNAs and indirectly regulates mRNA expression (19). IncRNA MEG3 previously was proved to have negative association with $m i R-494$ and $m i R-27 a$ in BC cell lines $(14,20)$. Thus, we explored the relationship between lncRNA $M E G 3$ and $m i R$ 93-5p. As expect, lncRNA $M E G 3$ negatively regulated the expression of $m i R-93-5 p$. Luciferase reporter assay further demonstrated that MiR-93-5p is a direct target of MEG3.

To confirm the regulatory mechanism of lncRNA $M E G 3$ and $m i R-93-5 p$ in BC progression, BC cells and xeno-graft mouse models with abnormal lncRNA MEG3 and miR-93$5 p$ expression were constructed. Either in vitro or in vivo, lncRNA MEG3 overexpression was showed to inhibit BC cell proliferation by decreasing the expression of proliferation markers (Ki67 and PCNA) and promote cell apoptosis by inhibiting pro-apoptotic proteins (cleaved caspase- 3 and caspase-9), while $m i R-93-5 p$ overexpression showed totally opposite results and promoted tumorigenesis. These results indicated that lncRNA MEG3 may function as a tumor suppressor gene while miR-93-5p as an oncogene. Multiple previous evidences demonstrated that overexpression of lncRNA MEG3 could suppress cell proliferation, migration and invasion $(11,14,15)$, which were concordant with our present finding. More importantly, we observed that upregulation of $m i R-93-5 p$ completely reversed the MEG3 overexpression mediated-suppression on tumorigenesis in BC cell lines and xeno-graft mouse models. These results further confirmed that lncRNA $M E G 3$ regulated the $\mathrm{BC}$ progression by targeting miR-93-5p. IncRNAs acts as ceRNAs, which could affect the post-transcriptional regulation by competing with targeting miRNAs and interfering with miRNA pathways $(21,22)$. IncRNA $M E G 3$ as a ceRNA of $m i R-19 a$, $m i R-181$ and $m i R N A-664$, was respectively demonstrated to 

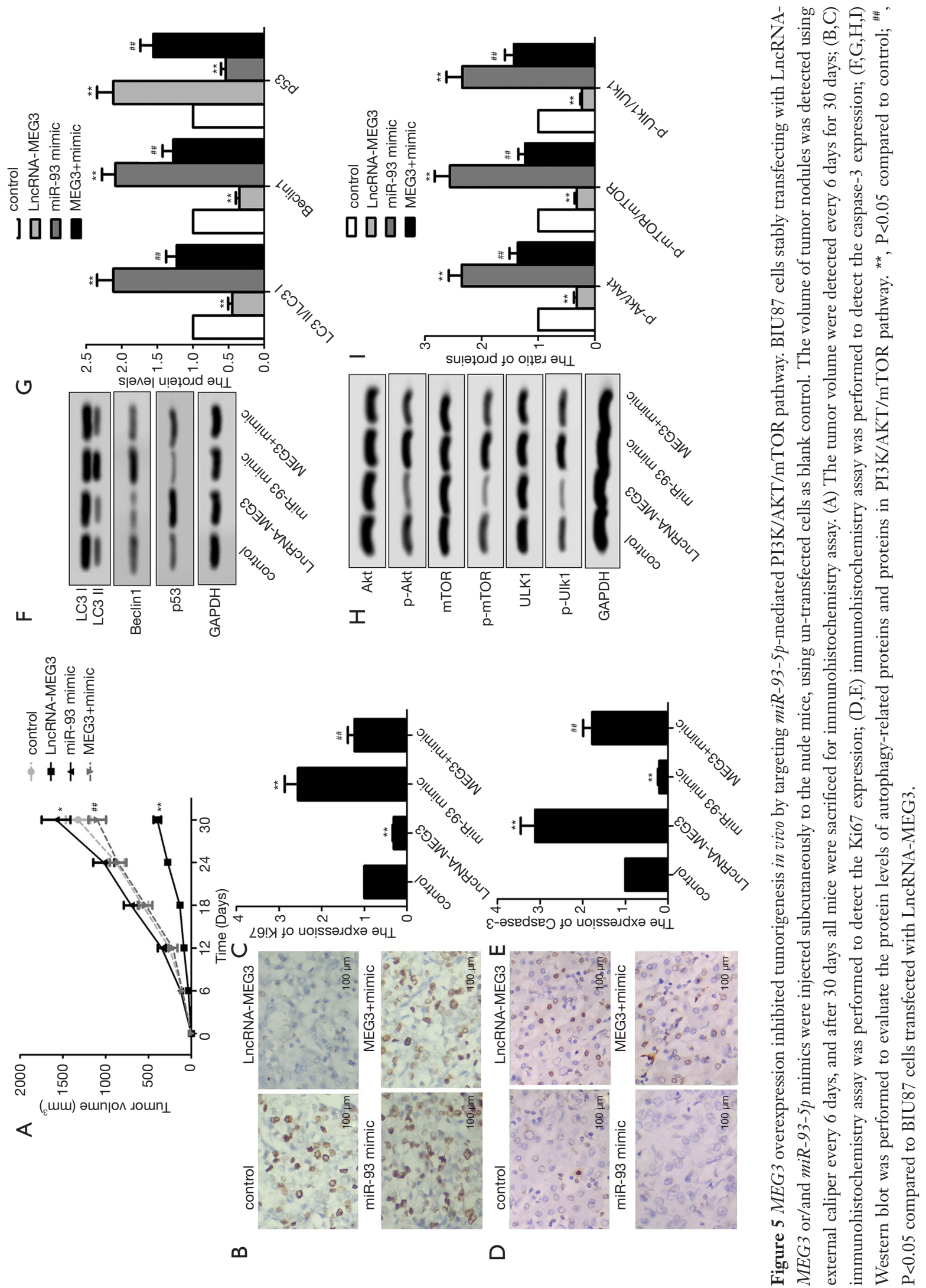
regulate the carcinogenesis in glioma (23), gastric cancer (7), hepatocellular cancer (24). Notably, lncRNA MEG3 was also reported to sponge $m i R-494$, thereby regulating the $\mathrm{BC}$ progression (14). Thus, we concluded that lncRNA $M E G 3$ could function as a ceRNA of $m i R-93-5 p$ to regulate the BC progression.

The miRNAs regulate the tumor growth through regulation of $\mathrm{PI} 3 \mathrm{~K} / \mathrm{AKT} / \mathrm{mTOR}$ pathway in various tumors (25). Among them, miR-93-5p was proved to promote metastasis and tumorigenesis of gliomas and nonsmall cell lung cancer by activating PI3K/AKT pathway $(12,26,27)$. The study showed overexpression of lncRNA $M E G 3$ decreased the rate of p-Akt/Akt, p-mTOR/mTOR and $\mathrm{p}-\mathrm{Ulk} / \mathrm{Ulk}$, while $m i R-93-5 p$ up-regulation reversed this effect. These results indicated that lncRNA $M E G 3$ can regulate the $\mathrm{PI} 3 \mathrm{~K} / \mathrm{AKT} / \mathrm{m}$ TOR pathway through sponging $m i R-93-5 p$. Additionally, we observed that overexpression of lncRNA $M E G 3$ could inhibit the autophagy-related proteins expression, which also be reversed by miR-93$5 p$ up-regulation. This demonstrated that up-regulation of lncRNA MEG3 can also inhibit the autophagy, thereby resulting the increase of cell apoptosis in BC. This finding was consistent with Ying et al. who reported downregulation of $M E G 3$ increased cell proliferation and activated autophagy of BC (9). In addition, in vivo assays of xeno-graft mouse model also validated that lncRNA $M E G 3$ could regulate autophagy and $\mathrm{PI} 3 \mathrm{~K} / \mathrm{AKT} / \mathrm{mTOR}$ pathway through sponging $m i R-93-5 p$ in the tumorigenesis of $\mathrm{BC}$.

\section{Conclusions}

Taken together, the present study demonstrated that IncRNA MEG3 functioned as a ceRNA of $m i R-93$ to suppress autophagy and induce cell apoptosis by inactivating $\mathrm{PI} 3 \mathrm{~K} / \mathrm{AKT} / \mathrm{m}$ TOR pathway in BC progression. The present research provided a new perspective to understanding the pathogenic mechanism of BC. The inhibition effect of $M E G 3$ on $\mathrm{BC}$ progression indicated that it may be a reliable diagnostic marker and effective therapeutic target for $\mathrm{BC}$.

\section{Acknowledgments}

Funding: None.

\section{Footnote}

Conflict of Interest: All authors have completed the ICMJE uniform disclosure form (available at http://dx.doi. org/10.21037/tcr.2020.01.70). The authors have no conflicts of interest to declare.

Ethical Statement: The authors are accountable for all aspects of the work in ensuring that questions related to the accuracy or integrity of any part of the work are appropriately investigated and resolved. The study was conducted in accordance with the Declaration of Helsinki (as revised in 2013). This study was approved by Ethics Committee of Peking Union Medical College Hospital (approval number: SYXK(Beijing)2018-0027). Informed consents were obtained from each patient before operation.

Open Access Statement: This is an Open Access article distributed in accordance with the Creative Commons Attribution-NonCommercial-NoDerivs 4.0 International License (CC BY-NC-ND 4.0), which permits the noncommercial replication and distribution of the article with the strict proviso that no changes or edits are made and the original work is properly cited (including links to both the formal publication through the relevant DOI and the license). See: https://creativecommons.org/licenses/by-nc-nd/4.0/.

\section{References}

1. Kamat AM, Hahn NM, Efstathiou JA, et al. Bladder cancer. Lancet 2016;388:2796-810.

2. Gislefoss RE, Stenehjem JS, Hektoen HH, et al. Vitamin $\mathrm{D}$, obesity and leptin in relation to bladder cancer incidence and survival: prospective protocol study. BMJ Open 2018;8:e019309.

3. Lv M, Zhong Z, Huang M, et al. lncRNA H19 regulates epithelial-mesenchymal transition and metastasis of bladder cancer by miR-29b-3p as competing endogenous RNA. Biochim Biophys Acta Mol Cell Res 2017;1864:1887-99.

4. Liu B, Sun L, Liu Q, et al. A cytoplasmic NF-kappaB interacting long noncoding RNA blocks IkappaB phosphorylation and suppresses breast cancer metastasis. Cancer Cell 2015;27:370-81.

5. Yin DD, Liu ZJ, Zhang E, et al. Decreased expression of long noncoding RNA MEG3 affects cell proliferation and predicts a poor prognosis in patients with colorectal cancer. Tumour Biol 2015;36:4851-9.

6. Ghafouri-Fard S, Taheri M. Maternally expressed gene 3 (MEG3): A tumor suppressor long non coding RNA. Biomed Pharmacother 2019;118:109129.

7. Peng W, Si S, Zhang Q, et al. Long non-coding RNA 
MEG3 functions as a competing endogenous RNA to regulate gastric cancer progression. J Exp Clin Cancer Res 2015;34:79.

8. Zhang X, Wu N, Wang J, et al. LncRNA MEG3 inhibits cell proliferation and induces apoptosis in laryngeal cancer via miR-23a/APAF-1 axis. J Cell Mol Med 2019;23:6708-19.

9. Ying L, Huang Y, Chen H, et al. Downregulated MEG3 activates autophagy and increases cell proliferation in bladder cancer. Mol Biosyst 2013;9: 407-11.

10. Tay Y, Rinn J, Pandolfi PP. The multilayered complexity of ceRNA crosstalk and competition. Nature 2014;505:344-52.

11. Huang C, Liao X, Jin H, et al. MEG3 as a competing endogenous RNA binds with miR-27a to promote PHLPP2 protein translation, and consequently impairs bladder cancer invasion. Cancer Res 2017;77:1898.

12. Zhang L, Liang X, Li Y. Long non-coding RNA MEG3 inhibits cell growth of gliomas by targeting miR93 and inactivating PI3K/AKT pathway. Oncol Rep 2017;38:2408-16.

13. Jacinto E, Werlen G. Mammalian Target of Rapamycin (mTOR). Compendium of Inflammatory Diseases 2016:874-92.

14. Shan G, Tang T, Xia Y, et al. MEG3 interacted with miR-494 to repress bladder cancer progression through targeting PTEN. J Cell Physiol 2020;235:1120-8.

15. Feng SQ, Zhang XY, Fan HT, et al. Up-regulation of LncRNA MEG3 inhibits cell migration and invasion and enhances cisplatin chemosensitivity in bladder cancer cells. Neoplasma 2018;65:925-32.

16. Lv D, Sun R, Yu Q, et al. The long non-coding RNA maternally expressed gene 3 activates $\mathrm{p} 53$ and is downregulated in esophageal squamous cell cancer. Tumour Biol 2016. [Epub ahead of print].

17. Kruer TL, Dougherty SM, Reynolds L, et al. Expression of the lncRNA Maternally Expressed Gene 3 (MEG3) Contributes to the Control of Lung Cancer Cell Proliferation by the Rb Pathway. PLoS One

Cite this article as: Fan X, Huang H, Ji Z, Mao Q. Long noncoding RNA MEG3 functions as a competing endogenous RNA of miR-93 to regulate bladder cancer progression via $\mathrm{PI} 3 \mathrm{~K} /$ AKT/mTOR pathway. Transl Cancer Res 2020;9(3):1678-1688. doi: $10.21037 /$ tcr.2020.01.70 2016;11:e0166363.

18. Luo G, Wang M, Wu X, et al. Long Non-Coding RNA MEG3 Inhibits Cell Proliferation and Induces Apoptosis in Prostate Cancer. Cell Physiol Biochem 2015;37:2209-20.

19. Anastasiadou E, Jacob LS, Slack FJ. Non-coding RNA networks in cancer. Nat Rev Cancer 2018;18:5-18.

20. Huang C, Liao X, Jin H, et al. MEG3, as a Competing Endogenous RNA, Binds with miR-27a to Promote PHLPP2 Protein Translation and Impairs Bladder Cancer Invasion. Mol Ther Nucleic Acids 2019;16:51-62.

21. Paraskevopoulou MD, Hatzigeorgiou AG: Analyzing MiRNA-LncRNA Interactions. In: Feng Y, Zhang L. editors. Long Non-Coding RNAs: Methods and Protocols. New York: Springer, 2016:271-86

22. Militello G, Weirick T, John D, et al. Screening and validation of lncRNAs and circRNAs as miRNA sponges. Brief Bioinform 2017;18:780-8.

23. Qin N, Tong GF, Sun LW, et al. Long Noncoding RNA MEG3 Suppresses Glioma Cell Proliferation, Migration, and Invasion by Acting as a Competing Endogenous RNA of miR-19a. Oncol Res 2017;25:1471-8.

24. He JH, Han ZP, Liu JM, et al. Overexpression of Long Non-Coding RNA MEG3 Inhibits Proliferation of Hepatocellular Carcinoma Huh7 Cells via Negative Modulation of miRNA-664. J Cell Biochem 2017;118:3713-21.

25. Du J, Liu S, He J, et al. MicroRNA-451 regulates stemness of side population cells via $\mathrm{PI} 3 \mathrm{~K} / \mathrm{Akt} / \mathrm{mTOR}$ signaling pathway in multiple myeloma. Oncotarget 2015;6:14993-5007.

26. Li C, Lyu J, Meng QH. MiR-93 Promotes Tumorigenesis and Metastasis of Non-Small Cell Lung Cancer Cells by Activating the PI3K/Akt Pathway via Inhibition of LKB1/ PTEN/CDKN1A. J Cancer 2017;8:870-9.

27. Jiang L, Wang C, Lei F, et al. miR-93 promotes cell proliferation in gliomas through activation of PI3K/Akt signaling pathway. Oncotarget 2015;6:8286. 